\title{
Introduction to 'The Unacceptable' Special Issue
}

\section{John Scannell, Macquarie University}

This special issue of Portal Journal of Multidisciplinary International Studies contends with the 'unacceptable' as the intervention upon bodies, images or practices deemed excessive to the limits of functional community. Under the rubric of propriety, discussion of the 'unacceptable' can all too easily become marginalised through silence, erasure and/or condemnation. Yet challenging the boundaries of the 'unacceptable' is vital to the continuity of a civil society, and the articles contained in this issue attempt to probe the limits of acceptability, as they seek to comprehend, and perhaps, intervene upon some divisive contemporary issues. Torture, disability, sexuality, e-waste, bureaucracy, comedy, and the constitution of the strange are among the broad range of topics in which the definition, regulation and assessment of unacceptability are pursued. Through documentation of such suitably 'unacceptable' issues, the articles contained in this volume not only compel the reader to question convention, but furthermore, interrogate the point where social intervention upon desire might be necessary too.

PORTAL Journal of Multidisciplinary International Studies, vol. 11, no. 2, July 2014.

The Unacceptable Special Issue, guest edited by John Scannell. 